\title{
Three new additions to the grass (Poaceae) flora of Manipur, India
}

\author{
Kangjam Tilotama Devi ${ }^{1}$, Potsangbam Kumar Singh ${ }^{2}$ and Debjyoti Bhattacharyya ${ }^{1^{*}}$ \\ ${ }^{1}$ Department of Life Science \& Bioinformatics, Assam University, Silchar 788 011, Assam, India \\ ${ }^{2}$ Department of Life Sciences, Manipur University, Canchipur, Imphal 795 003, Manipur, India
}

Article history

Received: 15 May 2016

Accepted: 16 July 2016

Published: 21 July 2016

(C) Devi et al. (2016)

Editor

K K Sabu

Publisher

Horizon e-Publishing Group

Corresponding Author

Dr. Debjyoti Bhattacharyya

d dbhattacharyya aus@yahoo.in

\begin{abstract}
Three grass species viz., Avena fatua L., Chrysopogon zizanioides (L.) Roberty and Digitaria violascens Link (Poaceae, nom. alt. Gramineae) are reported here for the first time from Manipur (India) as new records to the state. A key to the identification of species along with detail description and illustrations is provided to facilitate their easy identification.
\end{abstract}

\section{Keywords \\ Avena fatua; Chrysopogon zizanioides; Digitaria violascens; Gramineae; New Records}

Devi, K. T., P. K. Singh and D. Bhattacharyya. 2016. Three new additions to the grass (Poaceae) flora of Manipur, India. Plant Science Today 3(3): 272-281. http://dx.doi.org/10.14719/pst.2016.3.3.237

\section{Introduction}

The state Manipur, lying between $23^{\circ} 80^{\prime}-25^{0} 68^{\prime} \mathrm{N}$ latitude and $93^{\circ} 03^{\prime}-94^{\circ} 78^{\prime} \mathrm{E}$ longitude, is one of the eight states of North-Eastern India. The state shares international boundary with Myanmar and national border with Assam, Mizoram and Nagaland. The total geographical area is 22,327 sq. $\mathrm{km}$ of which about $90 \%$ is occupied by hilly regions and rest $10 \%$ by valley at the centre surrounded by hills. The state is divided into nine districts; among these, four districts viz., Bishnupur (Bishenpur), Imphal East, Imphal West and Thoubal are valley districts which are bounded by five hill districts viz. Chandel, Churachandpur, Senapati, Tamenglong and Ukhrul. A small part of Imphal East district (Jiribam sub-division) is lying adjacent to Barak Valley (Southern part of Assam). The altitudes of the state vary from $746 \mathrm{~m}$ to $1,583 \mathrm{~m}$ above msl.

In an ongoing study on the grass (Poaceae) flora of valley districts of Manipur, three new records have already been made by Devi et al.
(2014, 2015) recently. Another three species proclaim their first occurrence in the state after further exploration and investigation in the study area. These are: Avena fatua L., Chrysopogon zizanioides (L.) Roberty and Digitaria violascens Link. Hence, these reports constitute new records of these species from the state Manipur.

\section{Materials and Methods}

The present study is based on conventional methods of morpho-taxonomic research which include collection of fresh plant materials from the study area, preservation of specimens, critical morpho-taxonomic investigation, herbaria and literature survey for identification of species. Extensive and intensive field surveys were carried out for the collection of specimens from different localities of the study area during 2011-2015. Collection and preservation of specimens were done following routine herbarium practices recommended by Smith (1971), Jain \& Rao (1977), 

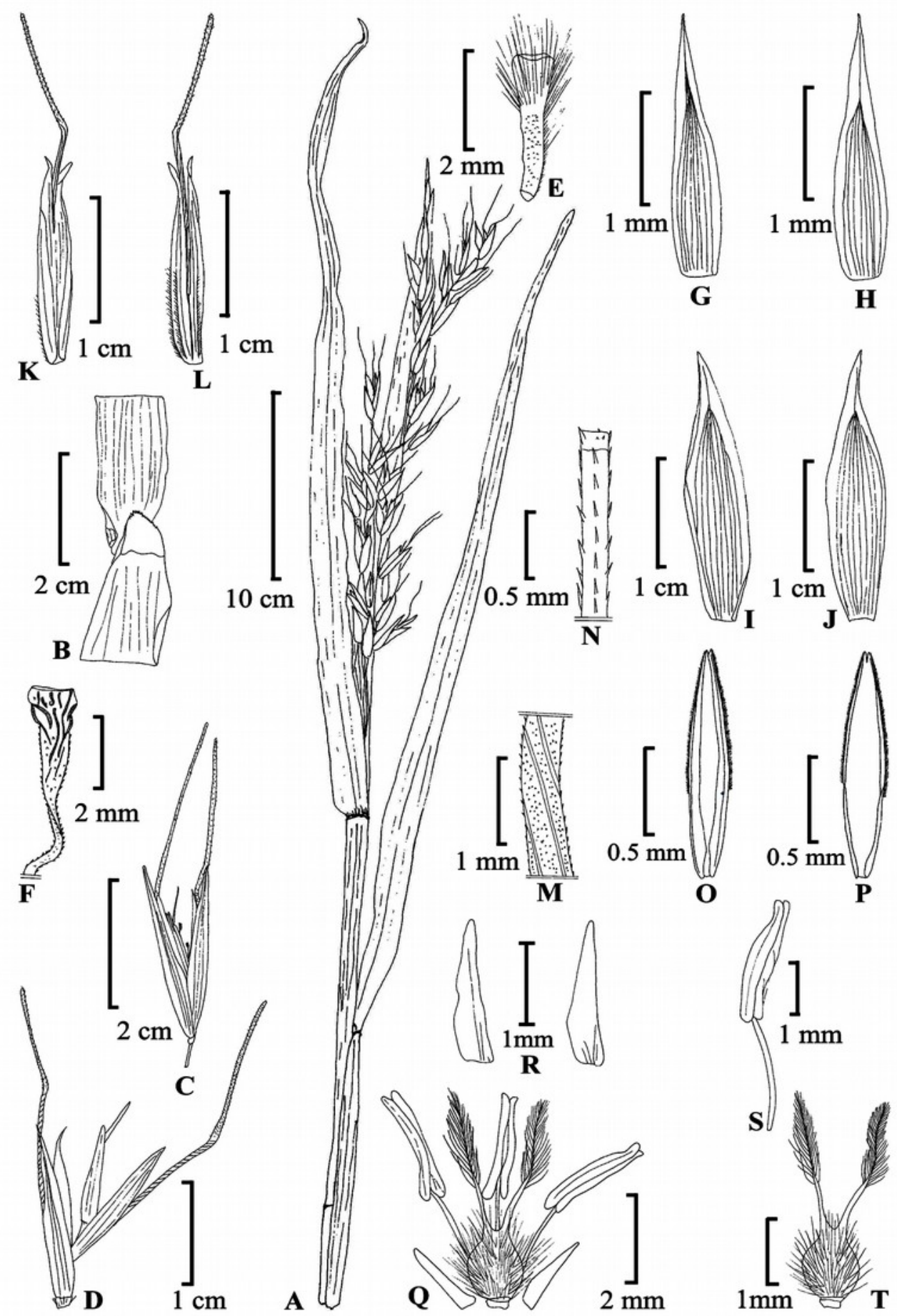

Fig. 1. Avena fatua L.: A. Habit; B. Ligule; C. Spikelet; D. Florets; E. Rachilla; F. Pedicel; G. Lower glume (Adaxial); H. Lower Glume (Abaxial); I. Upper Glume (Adaxial); J. Upper Glume (Abaxial); K. Lemma with awn (Adaxial); L. Lemma with awn (Abaxial); M. Column; N. Bristle; O. Palea (Adaxial); P. Palea (Abaxial); Q. Stamens and pistil with lodicules; R. Lodicules; S. Stamen; T. Pistil.

Van Balgooy (1987), Rao and Sharma (1990) and Singh \& Subramaniam (2008). All the specimens were critically examined under Olympus SZ61 stereo zoom dissecting microscope and finer structures were studied under Labomed Vision 2000 compound microscope. The taxa were identified after consultation of relevant taxonomic keys available in standard Floras, monographs and revisions (Bor 1940, 1960; Sreekumar and Nair 1991; Shukla 1996; Moulik 1997; Veldkamp 1999;
Kabeer and Nair 2009) and matching of specimens with the other authentic specimens housed in ASSAM and CAL (herbaria acronyms according to Thiers 2015). Digital images of specimens including type materials available at different herbarium databases (http://www.gbif.org, https://science.mnhn.fr, http://apps.kew.org/herbcat, https://plants.jstor.org, $\quad$ http://linneanonline.org/linnaean_herbarium.html) were consulted too for authentication of the identity of species. 


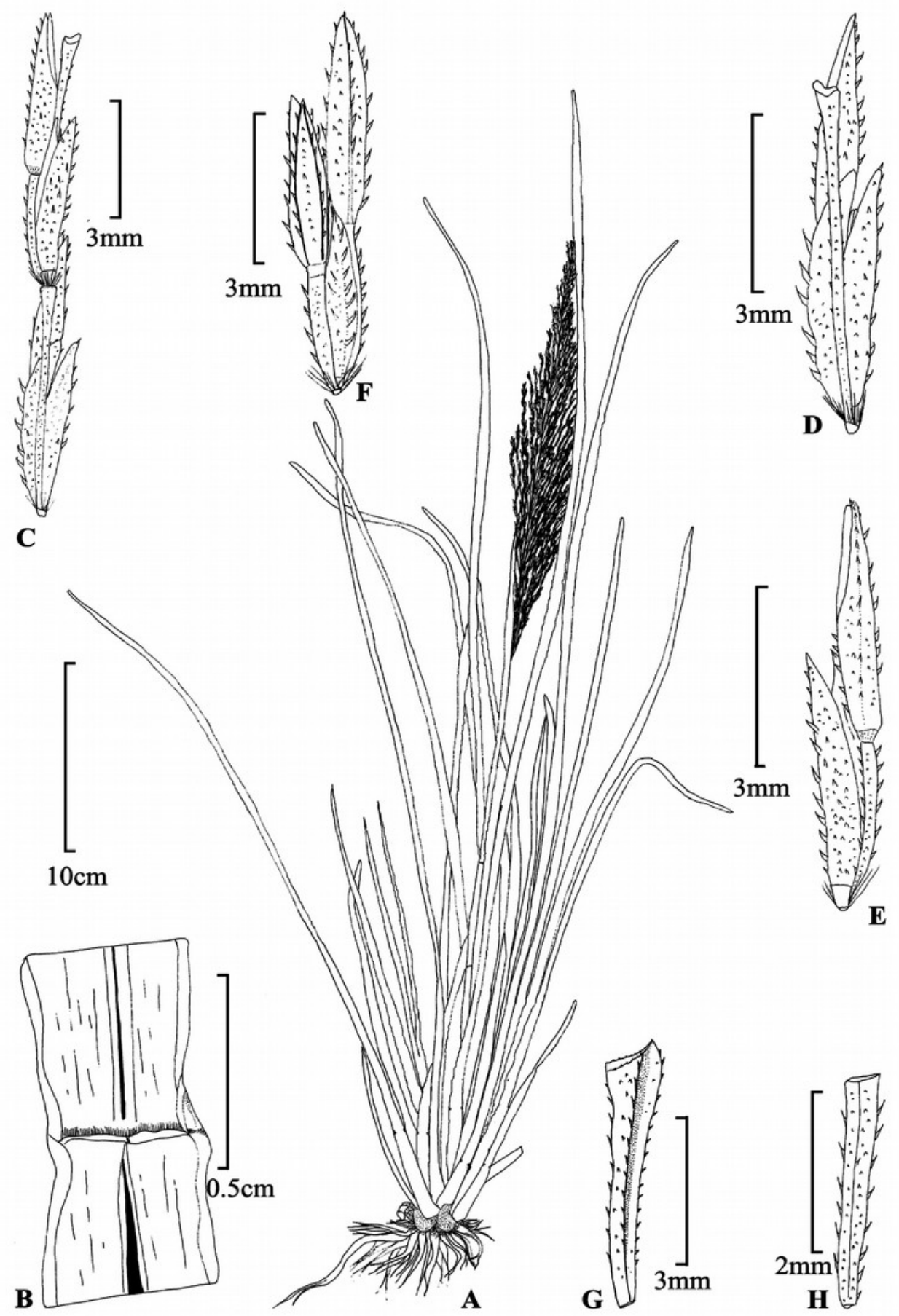

Fig. 2. Chrysopogon zizanioides (L.) Roberty: A. Habit; B. Ligule; C. Part of Raceme; D. Paired spikelets showing rachis internode; E. Paired spikelets showing pedicel; F. Terminal triad spikelets; G. Rachis; H. Pedicel.

A key based on morpho-taxonomic characters, detailed description with updated nomenclature and illustration of three species are provided here to facilitate their easy identification. The voucher specimens have been deposited in the Herbarium of Department of Life Science \& Bioinformatics, Assam University, Silchar with duplicate copies at ASSAM and CAL.

\section{Results and Discussion Taxonomic treatment}

Key to the species:

1. Racemes with nodding spikelets; spikelets 2-3.2 $\mathrm{cm}$, disarticulating above glumes and in between florets; florets $2-4$ 1. Avena fatua

1. Racemes without nodding spikelets; spikelets up to $6 \mathrm{~mm}$, disarticulating spikelets from pedicel, not above glumes and in between florets; florets 2 ....... 2 

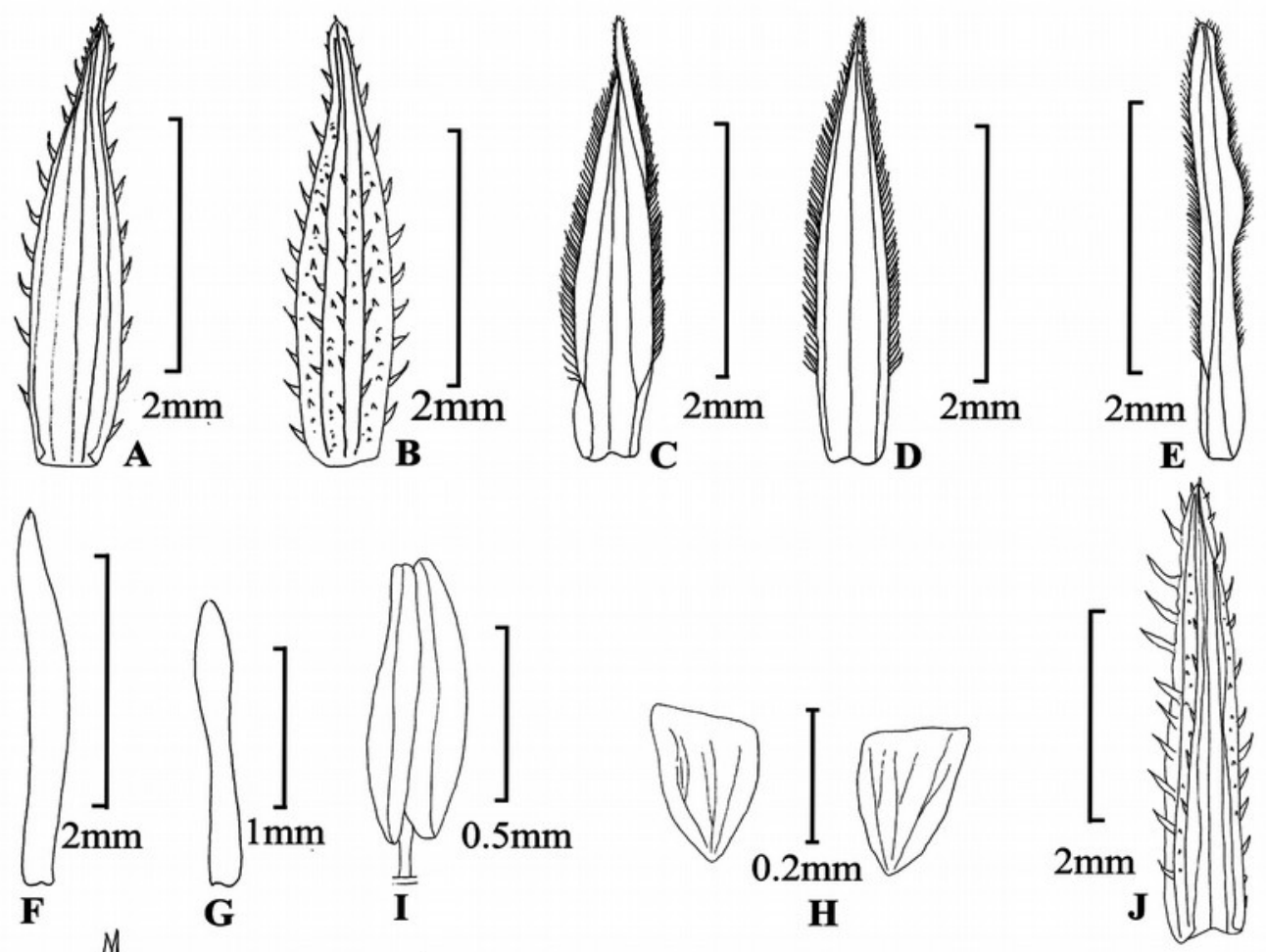

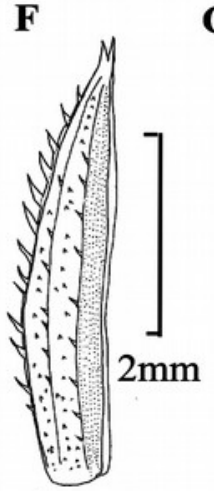

$\mathbf{L}$

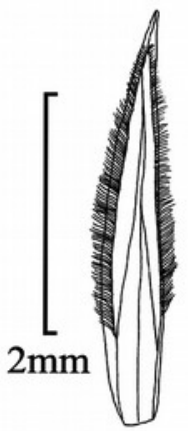

M

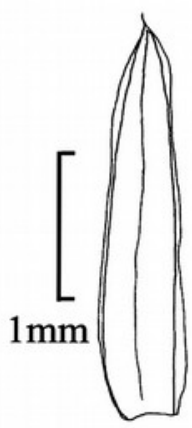

$\mathbf{N}$
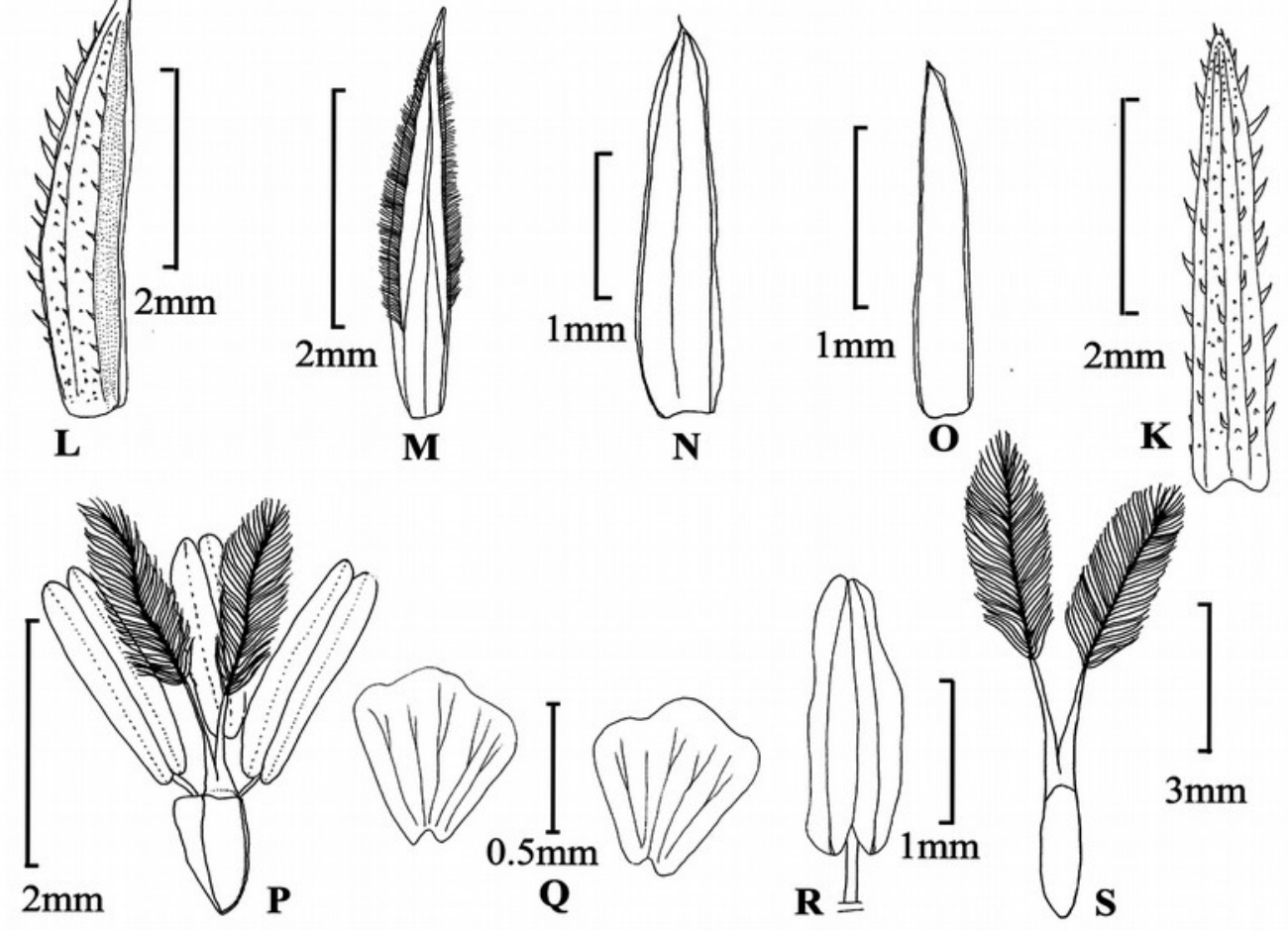

Fig. 3. Chrysopogon zizanioides (L.) Roberty: A - I (Pedicelled Spikelet): A. Lower glume (Adaxial); B. Lower Glume (Abaxial); C. Upper Glume (Adaxial); D. Upper Glume (Abaxial); E. Lower Lemma F. Upper Lemma; G. Upper Palea; H. Lodicules; I. Stamen. J - S (Sessile \& Fertile Spikelet): J. Lower glume (Adaxial); K. Lower Glume (Abaxial);

L. Upper Glume; M. Lower Lemma; N. Upper Lemma; O. Upper Palea; P. Stamens and pistil with lodicules; Q. Lodicules; R. Stamen; S. Pistil.

2. Racemes whorled; spikelets in pairs with a terminal triad; fertile spikelets $3.8-4.8 \mathrm{~mm}$ long, aculeate, muricate to spinulose

$$
\text { 2. Chrysopogon zizanioides }
$$

2. Racemes a digitate to subdigitate; spikelets ternate at least at middle, $1.5-2 \mathrm{~mm}$ long, fine appressed pubescent
1. Avena fatua L., Sp. Pl. 1: 80. 1753. Bor, Fl. Assam 5: 130.1940; Bor, Grasses Burma, Ceylon, India \& Pakistan: 434. 960; Karthik. et. al. Fl. Ind. Enum. Monocot.: 187. 1989. U. Shukla, Grasses N. E. India: 159. 1996; S. Moulik, Grasses Bamboos India 2: 439. 1997; Noltie, Fl. Bhutan 3(2): 585. t. 


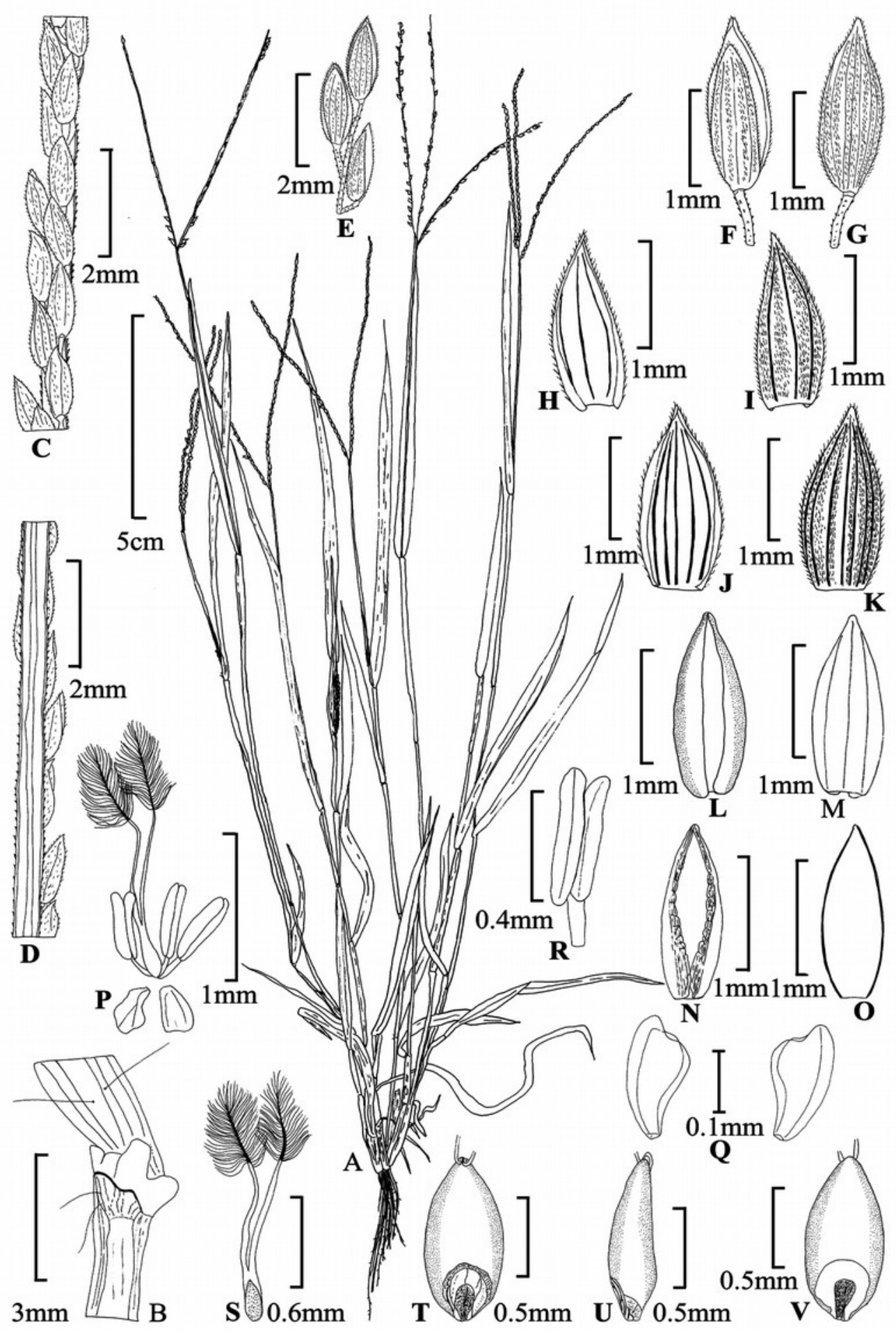

Fig. 4. Digitaria violascens Link: A. Habit; B. Ligule; C. \& D. Arrangement of spikelets on rachis; E. Spikelets in ternate F. Spikelet showing upper glume; G. Spikelet showing lower lemma; H. Upper Glume (Adaxial); I. Upper Glume (Abaxial); J. Lower Lemma (Adaxial); K. Lower Lemma (Abaxial); L. Upper Lemma (Adaxial); M. Upper Lemma (Abaxial); N. Upper Palea (Adaxial); O. Upper Palea (Abaxial); P. Stamens and pistil with lodicules; Q. Lodicules; R. Stamen; S. Pistil; T. Caryopsis showing embryo (Abaxial); U. Caryopsis (lateral view); V. Caryopsis showing hilum (Adaxial).

22(g-h). 2000; Kabeer \& V. J. Nair, Fl. Tamil Nadu Grasses: 78. 2009. Avena sativa subsp. fatua (L.) Thell., Vierteljahrsschr. Naturf. Ges. Zürich 56: 319 1912. Avena sativa var. fatua (L.) Fiori, Nuov. Fl. Italia 1: 109. 1923. - Type: PORTUGAL, Villa Franca, 06.1879, Cunha, A. Ricardo da, s.n. [Holotype COI
(COI00009264), digital image!]. Avena fatua L. subsp. glabrata (Peterm.) Piper \& Beattie, Fl. N.W. Coast 46. 1915. Avena fatua var. glabrata Peterm., Fl. Bienitz: 13.1841. - Type: PORTUGAL, Leziria d' Azambuja, s.d., Cunha, A. Ricardo da, s.n. [Holotype COI (COI00009268), digital image!]. 

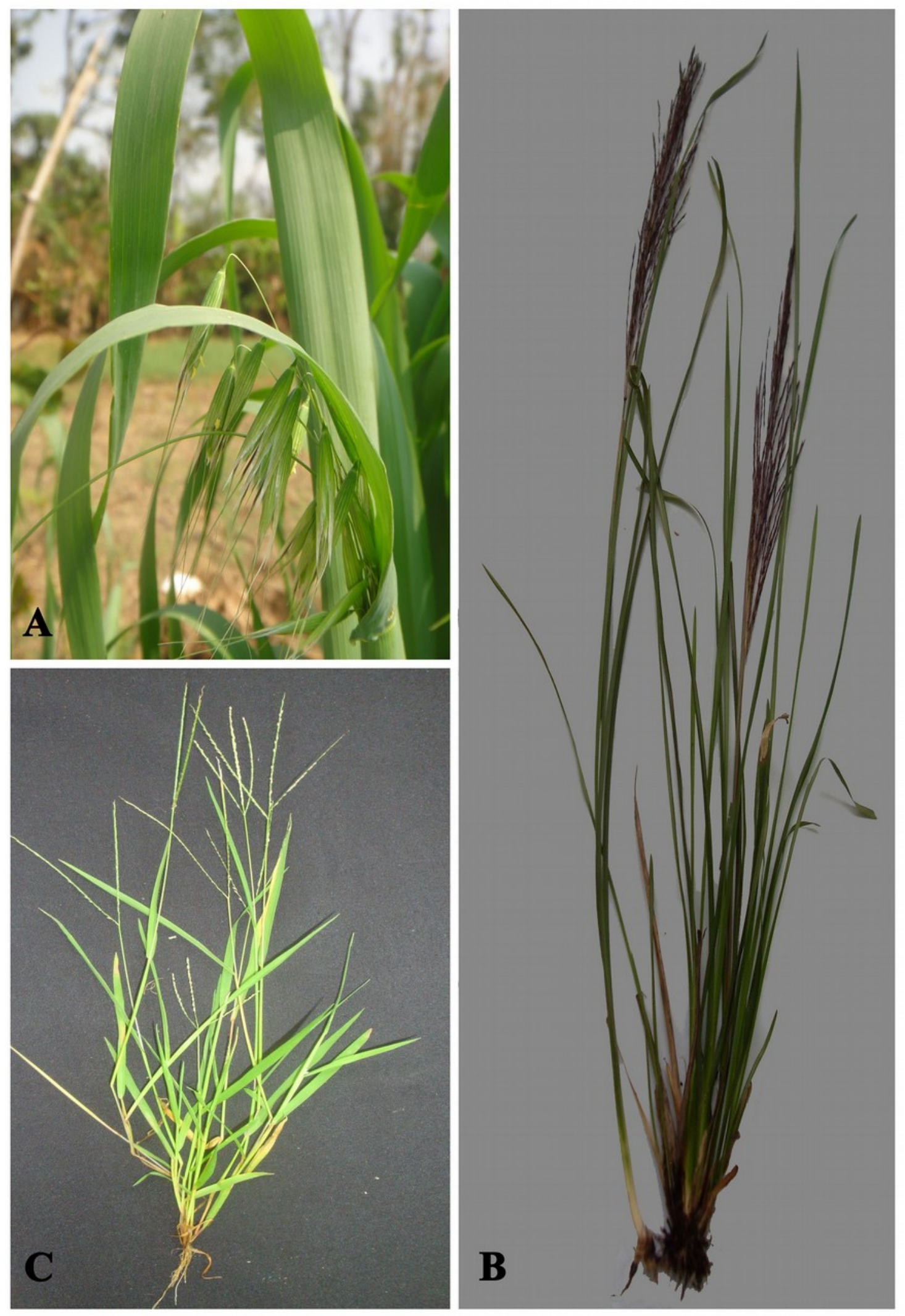

Fig. 5. Field photographs: A. Avena fatua; B. Chrysopogon zizanioides; C. Digitaria violascens.

Avena sativa L. var. sericea Hook.f., Fl. Brit. India 7: 275. 1896. - Type: INDIA, Sikkim, s.d., Hook. $f$. s.n. [Holotype, n.v.]; INDIA, Sikkim, 07.09.1849, Hook. f. s.n. [Lectotype K (K000032256), digital image!]; BHUTAN, Sikkim, s.d., Griffith, W. 2688, [Paralectotype K (K000032255), digital image!]. (Figs.1, 5A).
Vernacular Name: Not known. Wild Oat (English) An annual or a short-lived perennial, caespitose. Culms 50-110 cm long, erect to geniculate, more or less ribbed, herbaceous; nodes 3-5, constricted, brownish, sparsely pubescent to glabrous, sometimes retrorsely hairy below node. Leaves linear-lanceolate, $20-55 \times 0.7-2 \mathrm{~cm}$, rounded to 


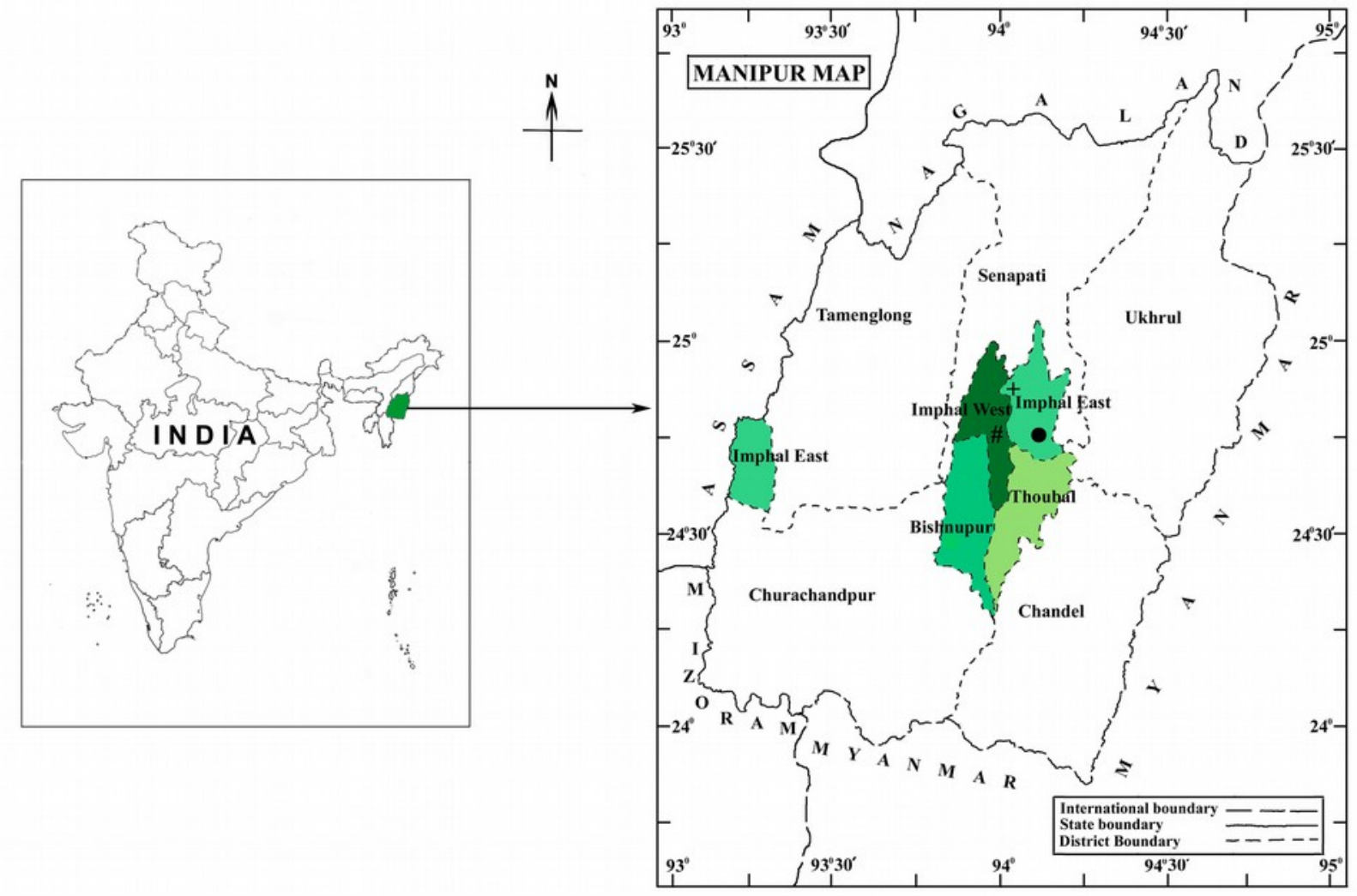

Map 1. Map of Manipur showing distribution of species in the study area [Avena fatua (•); Chrysopogon zizanioides (+); Digitaria violascens (\#)]

truncate at base, acuminate at apex, flat, scaberulous on both surfaces, serrulate at margin; midrib distinct, yellowish green; leaf sheaths 12-22 $\mathrm{x}$ 0.7-1 cm, slightly loose, more or less ribbed, sparsely long hairy at mouth when young, glabrous to finely tuberculate hairy at margin; ligules 3-8 mm, membranous, acute to obtuse at apex, whitish to hyaline, finely lacerate. Inflorescence a pyramidal panicle, 15-57 x 2.5-8 $\mathrm{cm}$, branches whorl at base, pale green to yellowish green; racemes 5-15 mm long, spreading with long pedicelled nodding spikelets; peduncle enclosed by sheath, more or less ribbed, barbellate; rachis rounded, scabrid; pedicels 1-6 x $0.2-0.3 \mathrm{~mm}$, linear to filiform, rigid, scabrid, serrate to baebellate at margins, mouth ca $1.2 \mathrm{~mm}$ wide. Spikelet solitary, nodding, laterally compressed, elliptic-lanceolate when young, 2-3.2 x $0.3-1 \mathrm{~cm}$, rounded at base, acuminate at apex, divergent and ovate when mature, pale green to yellowish green. Glumes similar and as long as spikelets, longer and enclosing florets, divergent at maturity, persistent. Lower glume ellipticlanceolate, $18-3.1 \times 0.4-0.8 \mathrm{~cm}$, rounded at base, acuminate at apex, flat, chartaceous, glabrous, 7-9nerved, distinct, anastomosing like a band, hyaline at margins, yellowish green. Upper glume ellipticlanceolate, $19-3.2 \times 0.4-0.9 \mathrm{~cm}$, rounded at base, acuminate at apex, chartaceous, flat, glabrous, 9- 10-nerved, distinct, anastomosing like a band, hyaline at margins, yellowish green. Florets $2-4$, similar, paleate, bisexual, sometimes $3^{\text {rd }}$ and $4^{\text {th }}$ florets reduced; disarticulating above glumes and in between florets; rachilla $2.5-4 \times 0.5-1.2 \mathrm{~mm}$, flat, solid, hispidulous, villous at one margin; hairs 0.8-2.2 mm long, yellowish green; callus bearded; hairs 1-2.3 mm long, whitish to rufous brown. Lemma elliptic-lanceolate, concave abaxially, the lower 10-20 x 1.3-5.8 $\mathrm{mm}$, the upper ones gradually reducing in size, gradually tapering towards apex, bi- to tetra-dentate with irregular lobes at apex, sub-coriaceous, 7-nerved, distinct, anastomosing like a band, hidpidulous, sparingly villous below middle; hairs $1.5-2 \mathrm{~mm}$ long, awned from middle, yellowish green; awn $2.5-4.7 \mathrm{~cm}$ overall, arising abaxially from the middle, geniculate; column $14.5-20 \times 0.4-0.5 \mathrm{~mm}$, twisted above, scabrid, brown; bristle 10.7-27 x 0.2-0.3 $\mathrm{mm}$, barbellate, yellowish green. Palea ellipticlanceolate, 8-17 x 2.2-3 mm, sub-coriaceous, rounded at base, shortly bi-dentate to emarginate at apex, incurved and glabrous at margins, sparsely ciliolate towards apex, 2-nerved, 2-keeled, yellowish green; keels wing and ciliolate. Lodicules linear to linear-lanceolate, $1.5-1.8 \times 0.2-0.4 \mathrm{~mm}$, obtuse at apex, whitish to hyaline. Stamens 3; filaments $2.3-2.6 \mathrm{~mm}$, hyaline; anthers $2-2.5 \times 0.3-$ $0.5 \mathrm{~mm}$, yellowish brown. Ovary broadly oblong, 
$0.7-1.5 \times 0.6-1.2 \mathrm{~mm}$, rounded to truncate at base, acute to truncate at apex, long rufous ciliate all over; hairs $0.5-2.7 \mathrm{~mm}$ long, extending beyond the style, yellowish brown; style 0.7-1 mm, hyaline; stigma 1.3-2.5 mm long, plumose, yellowish brown; stalk ca. $0.3 \mathrm{~mm}$. Caryopsis not seen.

\section{Flowering \& Fruiting: March - July.}

Habitat: Along the edge of cultivated field.

Distribution: INDIA (Arunachal Pradesh, Assam, Jammu-Kashmir, Manipur (present study), Meghalaya, Rajasthan, Sikkim, Tamil Nadu, Uttar Pradesh, West Bengal); Native to Europe, North Africa, North and South America, Australia and temperate Asia, now widely introduced and naturalized elsewhere.

Specimen Examined: INDIA, Manipur, Imphal East district, Poirou Khongjin, $24^{\circ} 40.986$ ' N, 94 $4^{\circ} 1.446^{\prime} \mathrm{E}$, 734 m, 15.03.2014, Coll.: K. Tilotama Devi 12061.

Uses: Fodder grass.

2. Chrysopogon zizanioides (L.) Roberty, Bull. Inst. Franc. Afrique Noire, A. 22: 106. 1960. Veldk., Austrobaileys 5: 522. 1999. Phalaris zizanioides L. Mant. Pl. Altera 183. 1771. Andropogon zizanioides (L.) Urb., Symb. Antill. 4: 79.1903. Vetiveria zizanioides (L.) Nash in Small, Fl. S.E. U.S. 67. 1903; Bor, Fl. Assam 5: 355.1940; Bor, Grasses Burma, Ceylon, India \& Pakistan: 258. 1960; Blatt. \& McCann, Bombay Grasses: 65. t. 40.1984; Karthik. et. al. Fl. Ind. Enum. Monocot.: 273. 1989; Sreek. \& V. J. Nair, Fl. Kerala Grasses: 203. 1991; U. Shukla, Grasses N. E. India: 145. 1996; S. Moulik, Grasses Bamboos India 1: 256. t. 51. 1997; Noltie, Fl. Bhutan 3(2): 790. t. 55(f-h). 2000; Kabeer \& V. J. Nair, Fl. Tamil Nadu Grasses: 488. 2009. - Type: s.l., s.d., s. coll., s.n. [Lectotype LINN (LINN-HL78-12), digital image!]. Andropogon muricatus Retz., Obs. Bot. 3: 43. 1783. - Type: s.l., s.d. Koenig, J.G., s.n. [Holotype LD n. v., Type (LD1228939), digital image!, fragm. K (K001050443), digital image!]. (Figs. 2, 3 \& 5B).

Vernacular Name: Charot (Manipuri); Vetiver (English).

A tussocky perennial. Roots aromatic, stout and fibrous. Culms 50-150(-200) cm high, caespitose, erect, unbranched, with a short rhizome, yellowish green; nodes a short furrowed ring, glabrous to puberulous, purplish to brownish; internodes 4-5, 6-13 cm long, more or less ribbed, glabrous; basal innovation intravaginal, flabellate. Leaf blade linear to linear-lanceolate, $24-85 \times 0.3-1 \mathrm{~cm}$, truncate at base, acute at apex, conduplicate towards base, flat towards apex, continuous from sheath, no clear deamarcation zone between leaf blade and sheath, midnerve distinct, slender and keeled, glabrous but pilose adaxially towards base, scabrous at margins, pale green; ligule a scarious rim, 0.1-0.2 mm long, with a fringe of 0.2-0.4 mm long white cilia; leaf sheath 5-12 x 0.3-1 cm, open, slender, spongy, glabrous, compressed, midnerve distinct and canal-like, nerves tessellated, more or less ribbed, margins flat, especially the lower ones terete, keeled, imbricate and flabellate. Inflorescence an open panicle, lanceolate to oblong, 18-27 x 1.3-6 cm, effuse to compact, racemes many, racemes whorled at nodes, purplish; inflorescence main axis internode shorter near base, longer towards apex; peduncle stout, 15-33 cm long, more or less ribbed, glabrous, straight, enclosed by sheath; racemes $3-12 \mathrm{~cm}$ long, suberect, appressed with 4-13 spikelet pairs with a terminal triad per raceme; raceme peduncle filiform, 1-3.5 cm long, smooth to scaberulous; rachis internode $3.5-5.8 \times 0.3-0.5 \mathrm{~mm}$, straight, triquetrous, turbinate, glabrous to setulose, cupuliform and ciliolate at the slightly oblique apex, alternate, purplish green; disarticulating with accessory parts. Spikelets paired, one sessile and fertile, another pedicelled and sterile; terminal triad with one sessile and 2 pedicelled spikelets, pale green to purplish; pedicel 2.5-3.2 $\mathrm{mm}$ long, more than half as long as the sessile spikelet, triquetrous, scaberulous, purplish green. Sessile spikelet linear-lanceolate to oblonglanceolate, slightly laterally compressed, 3.8-4.8 x 0.8-1.2 (including callus), aculeate, muricate to spinulose, pale green to purplish green; callus square, $0.3-0.8 \mathrm{~mm}$ long, rounded to obtuse at base, laterally ciliate at base, especially near the base of the rachis internode and pedicel; cilia 0.1$1.3 \mathrm{~mm}$ long, white, otherwise glabrous to puberulent. Lower glume lanceolate to oblonglanceolate, boat shaped, 3.5-4.3 x 0.5-0.7 mm, acute at apex, subcoriaceous, 3-7-nerved, 2-3 nerves distinct, lateral nerves very closed to each other and faint, 1-2-keeled, spinulose, aculeate and setulose, especially on nerves adaxially, pale green to purplish green. Upper glume lanceolate, sickle shaped, 3.5-4.3 x 0.5-0.7 mm, bifid and muticous at apex. subcoriaceous, chartaceous at near margins, 3-5-nerved, distinct, 1-keeled, spinulose and aculeate, especially on mid nerve, setulose adaxially, pale green to purplish green. Florets 2, lower barren, epaleate; upper hermaphrodite, paleate, without rachilla extension. Lower lemma ovate-.lanceolate to elliptic-lanceolate, 3.3-4 x 0.4$0.6 \mathrm{~mm}$, acute to acuminate at apex, membranous, hyaline, 3-nerved, faint, ciliolate at margins. Upper lemma ovate-lanceolate to elliptic-lanceolate, 2.3$3.2 \times 0.3-0.5 \mathrm{~mm}$, acute to acuminate with slightly bidentate at apex, membranous, hyaline, 3-nerved, faint, muticous to mucronate, awn from sinus, awn $0.4-3 \mathrm{~mm}$ long, straight to slightly geniculate, barbellate, column glabrous. Upper palea ellipticoblong to elliptic-lanceolate, $1.8-2.3 \times 0.2-0.5 \mathrm{~mm}$, acute to acuminate at apex, membranous, hyaline 2-nerved, faint. Lodicules 2, 0.5-0.8 x 0.4-0.7 mm, truncate at apex, membranous to chartaceous, hyaline to brownish. Stamens 3; filaments 0.7-1.5 $\mathrm{mm}$, hyaline; anthers 1.5-2 x 0.4-0.6 mm, brownish. Ovary elliptic to oblong-lanceolate, 0.5$1 \times 0.2-0.4 \mathrm{~mm}$, rounded to truncate at base, acute at apex, hyaline, greenish to brownish; style 0.5- 
$0.8 \mathrm{~mm}$ long, hyaline; stigma plumose, $0.8-1.6 \mathrm{~mm}$, greenish to brownish. Caryopsis not found. Pedicelled spikelets linear-lanceolate to oblonglanceolate, slightly laterally compressed, 3.2-4 x 0.5-1 mm, aculeate, muricate to spinulose, pale green to purplish green. Lower glume oblonglanceolate, $3.2-4 \times 0.6-0.8 \mathrm{~mm}$, acute to acuminate and muticous at apex, chartaceous to subcoriaceous, 5-nerved, 0-1-keeled, scaberulous, aculeate, especially on the nerves. Upper glume oblong-lanceolate, $3-3.8 \times 0.4-0.7 \mathrm{~mm}$, acute to acuminate and muticous at apex, chartaceous to subcoriaceous, 3-nerved, slightly 1-keeled at mid nerve, scaberulous and aculeate on midnerve, ciliolate at margins. Florets 2, lower barren, epaleate, upper staminate. Lower lemma lanceolate to oblong, 2.6-3.5 x 0.3-0.5 mm, similar with lower lemma of fertile spikelets. Upper lemma lanceolate to oblong, $2.5-3.2 \times 0.2-0.5 \mathrm{~mm}$, similar with upper lemma of fertile spikelets, mucronulate or not. Upper palea may be present or absent, if present, lanceolate to oblong, 1.5-2 x 0.2-0.4 mm, acute to truncate at apex, membranous, nerveless, hyaline. Lodicules 2, 0.2$0.3 \times 0.1-0.2 \mathrm{~mm}$, truncate at apex, membranous, hyaline. Stamens 3; filaments $0.6-0.8 \mathrm{~mm}$, hyaline; anthers $0.6-1.5 \times 0.3-0.4 \mathrm{~mm}$, brownish.

Flowering \& Fruiting: August - February

Habitat: Along the edges of streams and ponds; also common in marshy areas.

Distribution: INDIA (Assam, Bihar, Kerala, Madhya Pradesh, Maharashtra, Manipur (Present study), Meghalaya, Orissa, Tamil Nadu, Uttar Pradesh, West Bengal); Sri Lanka, Indo-China to Malaysia, South East Asia to Tropical Africa, introduced elsewhere.

Specimen Examined: INDIA, Manipur, Imphal East district, Haraorou Pangei, $24^{0} 54.854^{\prime}$ N, $93^{0} 58.827^{\prime}$ E, 787 m, 28.10.2015, Coll.: K. Tilotama Devi 12169.

Uses: Used as a fodder grass. Leaves and roots are used as one of the ingredients for the preparation of local hair care lotion called as Ching-hi.

3. Digitaria violascens Link, Hort. Berol. 1: 229. 1827. Bor, Fl. Assam 5: 208.1940; Bor, Grasses Burma, Ceylon, India \& Pakistan: 307. 1960; Karthik. et. al. Fl. Ind. Enum. Monocot.: 209. 1989; Sreek. \& V. J. Nair, Fl. Kerala Grasses: 242. 1991; U. Shukla, Grasses N. E. India: 322. 1996; S. Moulik, Grasses Bamboos India 1: 97. 1997; Noltie, Fl. Bhutan 3(2): 734. t. 45(c-e). 2000; Kabeer \& V. J. Nair, Fl. Tamil Nadu Grasses: 249. 2009. Panicum violascens (Link) Kunth, Révis. Gramin. 1: 33. 1829. - Type: BRAZIL, Brasilia, s.d., s. coll., Hb.Link.93? [Holotype B (B_10_0185495), digital image!]. (Figs. $4,5 \mathrm{C})$.

\section{Vernacular Name: Not known.}

Annuals or short lived perennials. Culms $20-70 \mathrm{~cm}$ high, caespitose, mat forming, decumbent to geniculately ascending, more or less ribbed, rhizomatous; nodes band like, glabrous, brownish; internodes 2-7 cm long; rooting from lower nodes. Leaf blade lanceolate to linear-lanceolate, flat, 3$27 \times 0.2-0.5 \mathrm{~cm}$, rounded to truncate at base, acuminate at apex, midnerve distinct, glabrous to sparsely pubescent on both sides, sparsely tuberculate hairy towards base adaxially, serrulate at margins; ligule a membrane, 1-1.4 mm long, obtuse to truncate at apex, entire to erose at margins, hyaline to brownish; leaf sheath 3-12 x 0.1-0.4 cm, closed, slender, midnerve distinct, terete above, keeled and distichous towards base, glabrous to sparsely pubescent, more or less ribbed, margins flat to incurved, mouth rarely bearded, falling later. Inflorescence composed of digitate to subdigitate racemes; peduncle $6-22 \mathrm{~cm}$ long, slender, more or less, capillary, more or less ribbed, enclosed by sheath; racemes (2-)3-6(-7), digitate to subdigitate, $3-12 \times 0.1-0.2 \mathrm{~cm}$ long, straight to curved, unilateral, pale green; rachis $0.5-0.8 \mathrm{~mm}$ wide, flat, broadly winged, straight to slightly flexuosus, finely serrulate at margins, ending with spikelets. Spikelets ternate at least at middle, otherwise paired, secund, pedicelled, homomorphous and fertile, elliptic-lanceolate to oblong-elliptic, $1.5-2 \times 0.5-0.8 \mathrm{~mm}$, truncate at base, acute at apex, pale green to whitish green due to fine appressed pubescent, disarticulating entire from pedicel; pedicels 2-3-nate, at middle third pedicel adnate with axis of rachis showing apparently single, unequal, 0.5-1.8 mm long, terete to angular, flat to triquetrous, solid, cupuliform at apex, scabrous. Lower glume absent. Upper glume ovate-elliptic to oblong-elliptic, $1.3-1.8 \times 0.4-0.6$ $\mathrm{mm}$, truncate at base, acute at apex, chartaceous, 3-5-nerved, distinct, appressed pubescent, hairs verrucose in between nerves and margins, pale green. Florets 2, lower barren, epaleate; upper hermaphrodite, paleate, without rachilla extension. Lower lemma elliptic-lanceolate to oblong-elliptic, $1.5-2 \times 0.5-0.8 \mathrm{~mm}$, rounded to truncate at base, acute to acuminate at apex, chartaceous, 5-7-nerved, appressed pubescent, hairs verrucose in between nerves and margins, sometimes glabrous flanking at midnerve interspaces, pale green. Upper lemma ellipticoblong to elliptic-lanceolate, boat shaped, 1.3-1.8 x $0.4-0.7 \mathrm{~mm}$, rounded to truncate at base, acute to acuminate at apex, subcoriaceous to cartilaginous, glossy, indurate, 3-nerved, faint, margins membranous, flat to involute covering most of palea, yellowish turning dark brown to blackish when matured. Upper palea elliptic-oblong to elliptic-lanceolate, $1.2-1.7 \times 0.4-0.6 \mathrm{~mm}$, truncate at base, acute to acuminate at apex, subcoriaceous to cartilaginous, glossy, indurate, 2-nerved, faint, margins membranous, flat to involute, yellowish turning dark brown to blackish when matured. Lodicules 2, $0.1-0.3 \times 0.1-0.2 \mathrm{~mm}$, cuneate to truncate at apex, membranous, hyaline, creamy to brownish. Stamens 3; filaments $0.2-0.5 \mathrm{~mm}$, hyaline; anthers $0.4-0.6 \times 0.1-0.2 \mathrm{~mm}$, yellowish to brownish. Ovary elliptic to oblong, $0.2-0.4 \times 0.1-$ $0.2 \mathrm{~mm}$, rounded to truncate at base, acute at apex, 
hyaline, greenish to brownish; style $0.5-0.7 \mathrm{~mm}$ long, hyaline; stigma plumose, $0.4-0.6 \mathrm{~mm}$, greenish to brownish. Caryopsis elliptic to oblong, 0.8-1.5 x 0.4-0.7 mm, subacute to obtuse at base, subacute to obtuse at apex, turgid, planoconvex, greenish to brownish green; embryo $0.3-0.5 \mathrm{~mm}$; hilum, $0.3-0.5 \mathrm{~mm}$.

\section{Flowering \& Fruiting: April - January}

Habitat: Along the road sides, forest margins, weedy and waste places.

Distribution: INDIA (Arunachal Pradesh, Assam, Karnataka, Kerala, Maharashtra, Manipur (Present study), Meghalaya, Nagaland, Orissa, Tamil Nadu, Uttar Pradesh, West Bengal); Tropical Asia, America, Africa and Australia, New Zealand, introduced elsewhere.

Specimen Examined: INDIA, Manipur, Imphal West district, Manipur University Campus, Canchipur, $24^{0} 45.27^{\prime} \mathrm{N}, 93^{0} 55.78^{\prime} \mathrm{E}, 726 \mathrm{~m}, 12.07 .2013$, Coll.: K. Tilotama Devi 11211.

Uses: Used as a fodder grass.

\section{Discussion}

The species enumerated here were hitherto unrecorded from Manipur as evidenced by literature survey (Deb 1961; Singh 1987; Karthikeyan et. al. 1989; Singh 1990; Shukla 1996; Moulik 1997; Khan et al. 2007; Barooah \& Ahmed 2014), so reported here as new records for the flora of Manipur. Map 1 shows the distribution of these species in the state; further exploration will add more information about their conservation status.

\section{Competing Interest}

The authors declare that they have no competing interests.

\section{Authors' contributions}

KTD carried out field explorations, worked out the specimens, prepared illustrations and drafted the manuscript. PKS and DB coordinated the work and interpreted the results. DB finalized the manuscript; all authors read and approved the final content of the manuscript. DB submitted the final script through his account.

\section{Acknowledgements}

The authors are thankful to the Head, Department of Life Science and Bioinformatics, Assam University, Silchar, for providing necessary facilities. They are thankful to the Additional Directors, Central National Herbarium (CAL), Botanical Survey of India, Howrah, and Eastern Regional Circle (ASSAM), Botanical Survey of India, Shillong, for permission to consult the herbarium and library. First author (KTD) thanks University Grants Commission, New Delhi, for financial assistance.

\section{References}

http://apps.kew.org/herbcat, accessed on 12.04. 2016.

http://linnean-online.org/linnaean_herbarium.html, accessed on 12.04. 2016.

https://plants.jstor.org, accessed on 12.04. 2016.

https://science.mnhn.fr, accessed on 12.04. 2016.

http://www.gbif.org, accessed on 12.04. 2016.

Barooah, C. and Ahmed, I. 2014. Plant diversity of Assam - a checklist of angiosperms \& gymnosperms. Assam Science Technology and Environment Council, Guwahati.

Bor, N. L. 1940. Gramineae in Kanjilal et al., Flora of Assam, Vol. 5, Government Press, Shillong.

Bor, N. L. 1960. The Grasses of Burma, Ceylon, India and Pakistan (excluding Bambuseae). Pergamon Press, London, Oxford, New York, Paris.

Deb, D. B. 1961. Monocotyledonous plants of Manipur Territory. Bull. Bot. Surv. India 3(2): 115 -138.

Devi, K.T., Singh, P.K and Bhattacharyya, D. 2014. Digitaria radicosa (J. Presl) Miq. \& Pennisetum purpureum Schumach.: Additions to the Grass (Poaceae) Flora of Manipur, India, J. Bombay Nat. Hist. Soc., 111(2): 152 153. doi: $10.17087 / j b n h s / 2014 / v 111 i 2 / 72254$

Devi, K.T., Singh, P.K and Bhattacharyya, D. 2015. Arthraxon hispidus (Thunberg) Makino [Poaceae]: a new record for the flora of Manipur, India, Pleione 9(1): 251 - 254.

Jain, S. K. and Rao, R. R. 1977. Handbook of Field and Herbarium methods. New Delhi.

Kabeer, K.A.A. and Nair, V.J. 2009. Flora of TamilNadu - Grasses. BSI. Kolkata.

Karthikeyan, S., Jain, S. K., M,. Nayar, P. and Sanjappa, M. 1989. Florae Indicae Enumeration: Monocotyledonae. BSI, Flora of India, Series 4.

Khan, M. R., Yadava, P. S, and Kikim, A. 2007. Additions to the flora of Manipur State. Bull. Bot. Surv. India. 49(1-4): 215 - 218.

Moulik, S. 1997. The Grasses and Bamboos of India. Vols. 1 \& 2. Scientific Publishers. Jodhpur.

Rao, R. R. and Sharma, B. D. 1990. A Manual for Herbarium Collections. BSI, Calcutta.

Shukla, U. 1996. The Grasses of North-Eastern India. Scientific Publishers. Jodhpur

Singh, H. B. and Subramaniam, B. 2008. Field Manual on Herbarium Techniques. National Institute of Science Communication and Information Resources (NISCAIR), CSIR, New Delhi. 298.

Singh, H. B. 1987. Floristic study of Tengnoupal District. Ph. D. Thesis submitted to the Manipur University.

Singh, O. K. 1990. Floristic study of Tamenglong District, Manipur with Ethnobotanical notes. Ph. D. Thesis submitted to the Manipur University.

Smith, C. Earle 1971. Preparing Herbarium specimens of Vascular Plants Agriculture Information Bulletin No. 348. Agricultural Research Service US Dept. of Agriculture.

Sreekumar, P. V. \& V. J. Nair 1991. Flora of Kerela - Grasses. BSI, Calcutta.

Thiers, B. 2015. Index Herbariorum: A global directory of public herbaria and associated staff. Available from: http://sweetgum.nybg.org/ih, accessed 15 July 2015.

Van Balgooy, M. M. J. 1987. A plant geographical analysis of Sulawesi. In: Whitmore, T. C. (ed.), Biogeographical Evolution of the Malay Archipelago. Oxford: Clarendon Press. 94-102.

Veldkamp, J. F. 1999. A Revision of Chrysopogon Trin. including Vetiveria Bory (Poaceae) in Thailand and Malesia with notes on some other species from Africa and Australia. Austrobaileya 5: 503 - 533.1999. 